\title{
A Homology Theory for Hybrid Systems: Hybrid Homology
}

\author{
Aaron D. Ames and Shankar Sastry \\ Department of Electrical Engineering and Computer Science, \\ University of California at Berkeley, \\ Berkeley, CA 94720 \\ \{adames, sastry\}@eecs.berkeley.edu
}

\begin{abstract}
By transferring the theory of hybrid systems to a categorical framework, it is possible to develop a homology theory for hybrid systems: hybrid homology. This is achieved by considering the underlying "space" of a hybrid system-its hybrid space or H-space. The homotopy colimit can be applied to this $\mathrm{H}$-space to obtain a single topological space; the hybrid homology of an H-space is the homology of this space. The result is a spectral sequence converging to the hybrid homology of an $\mathrm{H}$-space, providing a concrete way to compute this homology. Moreover, the hybrid homology of the H-space underlying a hybrid system gives useful information about the behavior of this system: the vanishing of the first hybrid homology of this H-space - when it is contractible and finite-implies that this hybrid system is not Zeno.
\end{abstract}

\section{Introduction}

In this paper we develop a homology theory for hybrid systems: hybrid homology. Up to this point, the limited mathematical understanding of hybrid systems has precluded the development of such a theory. In this paper, a categorical definition of a hybrid system is given; a hybrid system is essentially a small category $\mathfrak{H}$ of a specific form, called an $H$-small category, together with a functor from this small category to the category of dynamical systems: $\mathbf{S}_{\mathscr{H}}: \mathfrak{H} \rightarrow \mathfrak{D} \mathfrak{y n}$. This definition establishes a strong connection between the area of hybrid systems and the areas of algebraic topology and category theory. Preexisting mathematical constructions in these areas can be applied to hybrid systems when they are viewed from a categorical perspective.

The categorical approach to hybrid systems gives rise to the idea of the underlying "space" of a hybrid system: its hybrid space or $\mathrm{H}$-space, $\mathbb{H}$. An $\mathrm{H}$ space is given by an $\mathrm{H}$-small category $\mathfrak{H}$ and a functor, $\mathbf{S}_{\mathbb{H}}: \mathfrak{H} \rightarrow \mathfrak{T o p}$, from this small category to the category of topological spaces. Pairs of this formsmall categories together with functors to the category of topological spaceshave been well studied (cf. [1], [2, , 3]); important preexisting constructions can be applied to hybrid systems by exploiting this connection. A construction of special interest is the homotopy colimit which associates to an $\mathrm{H}$-space, $\mathbb{H}=\left(\mathfrak{H}, \mathbf{S}_{\mathbb{H}}\right)$, a 
single topological space, $\operatorname{Top}(\mathbb{H}):=\operatorname{hocolim}^{\mathfrak{H}}\left(\mathbf{S}_{\mathbb{H}}\right)$, referred to as the underlying topological space of the H-space $\mathbb{H}$.

The underlying topological space of an H-space allows us to define a homology theory of H-spaces simply by considering the homology of this space. This homology theory is termed hybrid homology and is denoted by $H H_{n}(\mathbb{H}, A):=$ $H_{n}(\operatorname{Top}(\mathbb{H}), A)$; here $A$ is an abelian group. One of the main impetuses for considering the homotopy colimit is that there is a spectral sequence converging to the homology of this space (cf. [1]). In the case of hybrid homology, this implies the existence of the hybrid homology spectral sequence

$$
E_{p, q}^{2}=H_{p}\left(\mathfrak{H}, H_{q}\left(\mathbf{S}_{\mathbb{H}}, A\right)\right) \Rightarrow H H_{p+q}(\mathbb{H}, A),
$$

where $H_{q}\left(\mathbf{S}_{\mathbb{H}}, A\right)$ is the functor from the small category $\mathfrak{H}$ to the category of abelian groups given by composing the homology functor with the functor $\mathbf{S}_{\mathbb{H}}$, and $H_{p}\left(\mathfrak{H}, H_{q}\left(\mathbf{S}_{\mathbb{H}}, A\right)\right)$ is the homology of the small category $\mathfrak{H}$ with coefficients in the functor $H_{q}\left(\mathbf{S}_{\mathbb{H}}, A\right)$. In this paper it will be seen that this spectral sequence gives very concrete ways to compute the hybrid homology of an H-space. Specifically, because of the particular structure of an H-small category, this spectral sequence reduces to a series of short exact sequences

$$
0 \longrightarrow H_{1}\left(\mathfrak{H}, H_{n-1}\left(\mathbf{S}_{\mathbb{H}}, A\right)\right) \longrightarrow H H_{n}(\mathbb{H}, A) \longrightarrow H_{0}\left(\mathfrak{H}, H_{n}\left(\mathbf{S}_{\mathbb{H}}, A\right)\right) \longrightarrow 0 .
$$

In the case when the $\mathrm{H}$-space $\mathbb{H}$ is contractible, i.e., when each domain of the $\mathrm{H}$-space is contractible, the spectral sequences collapses to yield isomorphisms

$$
H H_{n}(\mathbb{H}, A) \cong H_{n}(\mathfrak{H}, A),
$$

where $H_{n}(\mathfrak{H}, A)$ is the homology of the small category $\mathfrak{H}$ with coefficients in an abelian group $A$. The startling point is that these facts can be used to show that the hybrid homology of an H-space dictates the type of behavior that a hybrid system on this H-space can have, especially with regard to Zeno.

Given a (categorical) hybrid system $\mathscr{H}$ we can associate to it its underlying $H$-space $\mathbb{H}^{\mathscr{H}}$, and we are able to show that this space gives useful information about the hybrid system. By considering the forgetful functor $\mathbf{U}: \mathfrak{H} \mathfrak{c a t} \rightarrow \mathfrak{G r p h}$ from that category of H-small categories to the category of small graphs, we are able to show that in the case when $\mathbb{H}$ is contractible and finite there is an isomorphism

$$
H H_{n}(\mathbb{H}, \mathbb{R}) \cong H_{n}(\mathbf{U}(\mathfrak{H}), \mathbb{R}),
$$

where $H_{n}(\mathbf{U}(\mathfrak{H}), \mathbb{R})$ is the graph homology of the graph $\mathbf{U}(\mathfrak{H})$. By considering the underlying H-space $\mathbb{H}^{\mathscr{H}}$ of a hybrid system $\mathscr{H}$, this result together with the results of [4] allows us to show that when $\mathbb{H}^{\mathscr{H}}$ is contractible and finite

$$
\operatorname{dim}_{\mathbb{R}} H H_{1}\left(\mathbb{H}^{\mathscr{H}}, \mathbb{R}\right)=\operatorname{dim}_{\mathbb{R}} \mathcal{N}\left(K_{\mathbf{U}(\mathfrak{H})}\right)=0 \quad \Rightarrow \quad \mathscr{H} \text { is not Zeno }
$$

where $\mathcal{N}\left(K_{\mathbf{U}(\mathfrak{H})}\right)$ is the null space of the incidence matrix $K_{\mathbf{U}(\mathfrak{H})}$ of the graph $\mathbf{U}(\mathfrak{H})$. This final statement seems to imply that the definition of hybrid homology is the right one because it gives useful information about the hybrid system. The statement also supports the claim that the theory developed here has useful and practical implications. 


\section{Categorical Hybrid Systems}

Up to this point, a hybrid system has been defined to be a tuple which is a collection of spaces subject to certain relations given by maps between these spaces. A set of vector fields or flows is also included in the definition. To better understand hybrid systems, we consider this collection of spaces and this collection of flows separately; the former is referred to as a hybrid space or H-space and the latter is a "flow" on this H-space. The motivation for this is derived from dynamical system theory where there is a clear distinction between the "underlying topological space" of a dynamical system and a flow on that space. Paralleling dynamical systems, a hybrid system is obtained by adding a collection of flows to an H-space.

In this section, we give the classical definition of an H-space and then proceed to give a categorical definition of an H-space in terms of a small category and a functor; the advantage of the categorical definition of an $\mathrm{H}$-space is that it is not only more general but also more concise. We then proceed to give the definition of a hybrid system utilizing the categorical framework developed - a hybrid system is also defined by a small category and a functor. These constructions will be essential in developing a homology theory for hybrid systems, although this seems to be only the first step in exploring their power.

H-Space. Define a classical $H$-space (short for classical hybrid space) as a tuple

$$
\mathbb{H}_{\text {class }}=(Q, E, D, G, R)
$$

where

$-Q=\{1, \ldots, m\} \subset \mathbb{Z}$ is a set of discrete states.

$-E \subset Q \times Q$ is a set of edges which define relations between the domains. For $e=(i, j) \in E$, we denote the source of $e$ by $\mathfrak{s}(e)=i$ and the target of $e$ by $\mathfrak{t}(e)=j$.

- $D=\left\{D_{i}\right\}_{i \in Q}$ is a set of domains where $D_{i}$ is a topological space.

- $G=\left\{G_{e}\right\}_{e \in E}$ is a set of guards, where $G_{e} \subseteq D_{\mathfrak{s}(e)}$ is also a topological space.

- $R=\left\{R_{e}\right\}_{e \in E}$ is a set of reset maps or transition maps; these are continuous maps from $G_{e} \subseteq D_{\mathfrak{s}(e)}$ to $R_{e}\left(G_{e}\right) \subseteq D_{\mathfrak{t}(e)}$.

The subscript "class" indicates that this is a "classical" definition, meaning that this definition is one of the most commonly used ones (cf. [5]). For a classical Hspace the pair $(Q, E)$ is an oriented graph (technically a pseudograph), so we can write a classical H-space as a tuple $\mathbb{H}_{\text {class }}=(\Gamma, D, G, R)$. The graph $\Gamma=(Q, E)$ is referred to as the underlying graph of the H-space.

We can demand that the collection of topological spaces $D$ be a collection of manifolds $M=\left\{M_{i}\right\}_{i \in Q}$, that the maps $R$ be a collection of smooth embeddings $R^{S}$, and that the set of guards be a set of smooth manifolds, $G^{M}$, such that $G_{e}^{M}$ is an embedded submanifold of $M_{\mathfrak{s}(e)}$. In this case we call the H-space $\mathbb{G}_{\text {class }}=\left(Q, E, M, G^{M}, R^{S}\right)$ a smooth classical hybrid space or a smooth classical $H$-space or a classical $G$-space. This more restrictive definition is the starting point for much of the literature on hybrid systems. 
Example 1. The hybrid system modeling a water tank system (cf. [5] for a complete explanation, although we assume the reader is familiar with this example) is a classical example of a hybrid system that displays Zeno behavior. Beyond this observation, we will not discuss the dynamics of this hybrid system as in this paper we are more interested in its underlying "space". The hybrid space for the water tank will be denoted by $\mathbb{H}_{\text {class }}^{W}=\left(\Gamma^{W}, D^{W}, G^{W}, R^{W}\right)$. It has as its underlying graph $\Gamma_{W}$ given by the diagram

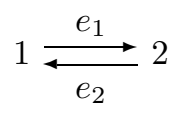

The other elements of the hybrid system are defined as: $D_{1}^{W}=D_{2}^{W}=\left\{\left(x_{1}, x_{2}\right)\right.$ : $\left.x_{1}, x_{2} \geq 0\right\}, G_{e_{1}}^{W}=\left\{\left(x_{1}, 0\right): x_{1} \geq 0\right\}, G_{e_{2}}^{W}=\left\{\left(0, x_{2}\right): x_{2} \geq 0\right\}$, and $R_{e_{1}}^{W}\left(x_{1}, x_{2}\right)=R_{e_{2}}^{W}\left(x_{1}, x_{2}\right)=\left(x_{1}, x_{2}\right)$. We will refer back to this example throughout this paper in order to illustrate the concepts being introduced.

H-Small Categories. An H-small category is a small category $\mathfrak{H}$ (cf. 6 for more information on small categories and category theory in general) satisfying the following conditions:

1. Every object in $\mathfrak{H}$ is either the source of a non-identity morphism in $\mathfrak{H}$ or the target of a non-identity morphism but never both, i.e., for every diagram

$$
a_{0} \stackrel{\alpha_{1}}{\longrightarrow} a_{1} \stackrel{\alpha_{2}}{\longrightarrow} \cdots \stackrel{\alpha_{n}}{\longrightarrow} a_{n}
$$

in $\mathfrak{H}$, all but one morphism must be the identity (the longest chain of composable non-identity morphisms is of length one).

2. If an object in $\mathfrak{H}$ is the source of a non-identity morphism, then it is the source of exactly two non-identity morphisms, i.e., for every diagram in $\mathfrak{H}$ of the form

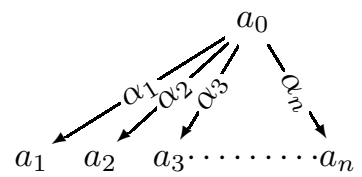

either all of the morphisms are the identity or two and only two morphisms are not the identity.

Important Objects in H-Small Categories. Let $\mathfrak{H}$ be an H-small category. We use $\mathfrak{O} \mathfrak{b}(\mathfrak{H})$ to denote the objects of $\mathfrak{H}$ and $\mathfrak{M o r}_{\mathrm{i} d}(\mathfrak{H})$ to denote the non-identity morphisms of $\mathfrak{H}$; all of the morphisms in $\mathfrak{H}$ are the union of these morphisms with the identity morphism from each object to itself. For a morphism $\alpha: a \rightarrow b$ in $\mathfrak{H}$, its source is denoted by $\mathfrak{s}(\alpha)=a$ and its target is denoted by $\mathfrak{t}(\alpha)=b$. For $\mathrm{H}$-small categories, there are two sets of objects that are of particular interest; these are subsets of the set $\mathfrak{O b}(\mathfrak{H})$. The first of these is called the wedge set, denoted by $\wedge(\mathfrak{H})$, and defined to be

$$
\wedge(\mathfrak{H}):=\left\{a \in \mathfrak{O} \mathfrak{b}(\mathfrak{H}): a=\mathfrak{s}(\alpha), \quad a=\mathfrak{s}(\beta), \quad \alpha, \beta \in \mathfrak{M o r}_{\mathrm{i}, d}(\mathfrak{H}), \quad \alpha \neq \beta\right\} .
$$


For all $a \in \wedge(\mathfrak{H})$ there are two and only two morphisms (which are not the identity) $\alpha, \beta \in \mathfrak{M o r}_{\mathrm{i}, d}(\mathfrak{H})$ such that $a=\mathfrak{s}(\alpha)$ and $a=\mathfrak{s}(\beta)$, so we denote these morphisms by $\alpha_{a}$ and $\beta_{a}$. Conversely, given a morphism $\gamma \in \mathfrak{H}$ (which is not the identity), there exists a unique $a \in \wedge(\mathfrak{H})$ such that $\gamma=\alpha_{a}$ or $\gamma=\beta_{a}$. The symbol $\wedge$ is used because every object $a \in \wedge(\mathfrak{H})$ sits in a diagram of the form:

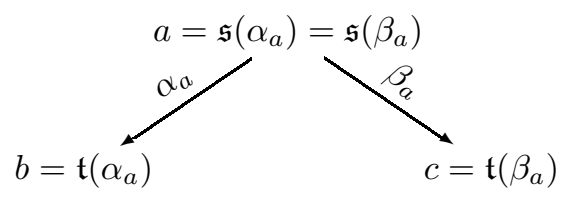

Note that giving all diagrams of this form (of which there is one for each $a \in$ $\wedge(\mathfrak{H}))$ gives all the objects in $\mathfrak{H}$, i.e., every object of $\mathfrak{H}$ is the target of $\alpha_{a}$ or $\beta_{a}$, or their source, for some $a \in \wedge(\mathfrak{H})$. In particular, we can define $\vee(\mathfrak{H})=(\wedge(\mathfrak{H}))^{c}$ where $(\wedge(\mathfrak{H}))^{c}$ is the complement of $\wedge(\mathfrak{H})$ in the set $\mathfrak{O} \mathfrak{b}(\mathfrak{H})$.

Definition 1. A categorical $H$-space is a pair $\mathbb{H}_{\text {cat }}=\left(\mathfrak{H}, \mathbf{S}_{\mathbb{H}}\right)$ where $\mathfrak{H}$ is an $H$-small category and $\mathbf{S}_{\mathbb{H}}: \mathfrak{H} \rightarrow$ Top is a functor such that for every diagram of the form

$$
A \stackrel{\alpha}{\stackrel{\beta}{\longrightarrow}} \stackrel{\beta}{\longrightarrow} B
$$

in $\mathfrak{H}$ in which $\alpha$ and $\beta$ are not the identity, either $\mathbf{S}_{\mathbb{H}}(\alpha)$ or $\mathbf{S}_{\mathbb{H}}(\beta)$ is an inclusion.

Theorem 1. There is an injective correspondence

$$
\left\{\text { Classical } \mathrm{H}-\text { spaces, } \mathbb{H}_{\text {class }}\right\} \longrightarrow\left\{\text { Categorical } \mathrm{H}-\text { spaces, } \mathbb{H}_{\text {cat }}\right\}
$$

This is a bijective correspondence if $\mathfrak{H}$ has a finite number of objects.

Example 2. The categorical hybrid space for the water tank, $\mathbb{H}_{\text {cat }}^{W}=\left(\mathfrak{H}^{W}, \mathbf{S}_{\mathbb{H}}^{W}\right)$ is defined by the following diagram:
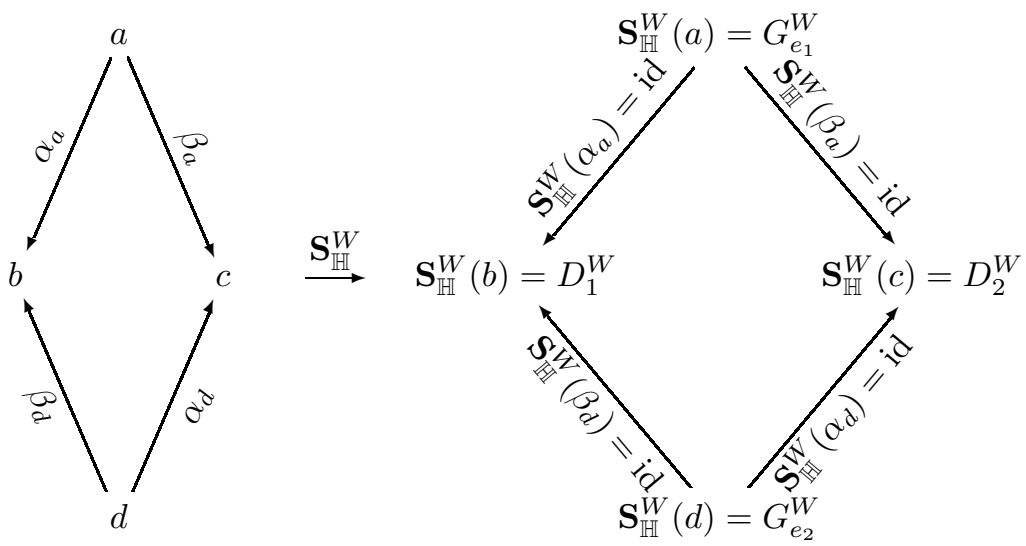

Note that the H-small category $\mathfrak{H}^{W}$ is defined by the diagram on the left together with the identity morphism on each object, while the functor $\mathbf{S}_{\mathbb{H}}^{W}$ is defined by 
the diagram of topological spaces on the right. To complete the description of the functor $\mathbf{S}_{\mathbb{H}}^{W}$, on identity morphisms $\mathbf{S}_{\mathbb{H}}^{W}$ is defined to be the identity map.

Smooth Categorical Hybrid Spaces. We can define a categorical G-space in a way analogous to the definition of a categorical H-space, i.e., it is a pair $\mathbb{G}_{\text {cat }}=\left(\mathfrak{H}, \mathbf{T}_{\mathbb{G}}\right)$, where $\mathfrak{H}$ is an H-small category and $\mathbf{T}_{\mathbb{G}}$ is a functor $\mathbf{T}_{\mathbb{G}}: \mathfrak{H} \rightarrow$ $\mathfrak{M a n}$ from $\mathfrak{H}$ to the category of manifolds, such that the pair $\left(\mathfrak{H}, \mathbf{I} \circ \mathbf{T}_{\mathbb{G}}\right)$ is also a categorical H-space; here I : Man $\rightarrow \mathfrak{T o p}$ is the inclusion functor. With this definition, Theorem 1 yields the following corollary.

Corollary 1. There is an injective correspondence

$$
\left\{\text { Classical G- spaces, } \mathbb{G}_{\text {class }}\right\} \longrightarrow\left\{\text { Categorical G- spaces, } \mathbb{G}_{\text {cat }}\right\}
$$

The Category of Dynamical Systems. We can consider both the category of dynamical systems and the category of smooth dynamical systems. The category of dynamical systems, denoted by $\mathfrak{D} \mathfrak{n} \mathfrak{n}$, has as objects dynamical systems and dynamical subsystems. A dynamical system is a pair $(X, \varphi)$ where $X$ is a topological space and $\varphi$ is a flow on that topological space - more precisely, this is a local flow (cf. [7]). A morphism of two dynamical systems $\alpha:(X, \varphi) \rightarrow(Y, \psi)$ in this category is defined by a pair $\alpha=(h, r)$ of continuous maps, $h: X \rightarrow Y$ and $r: \mathbb{R} \rightarrow \mathbb{R}$, such that the following diagram

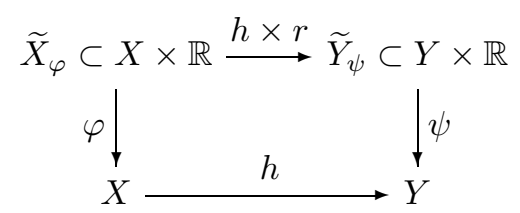

commutes, i.e., $h\left(\varphi_{t}(x)\right)=\psi_{r(t)}(h(x))$; here $\widetilde{X}_{\varphi}$ is the maximal flow domain of the flow $\varphi$ (as defined in [7]). Clearly, from this definition it follows that two dynamical systems are isomorphic (in the categorical sense) if and only if they are topologically orbital equivalent. A dynamical subsystem is a pair $\left(U \subseteq X,\left.\varphi\right|_{U}\right)$ where $U$ is a topological space contained in $X$ and $\left.\varphi\right|_{U}$ is the restriction of a flow $\varphi$ on $X$ to $U$; we say that this dynamical subsystem is a subsystem of $(X, \varphi)$. Morphisms of dynamical subsystems are defined in a way analogous to the definition of morphisms of dynamical systems (cf. [8] for a definition).

Similarly, we can define the category $\mathfrak{S} \mathfrak{d y n}$ of smooth dynamical systems whose objects are smooth dynamical systems and smooth dynamical subsystems 1 A smooth dynamical system is a pair $(M, V)$ where $M$ is a manifold and $V$ is a vector field on that manifold (both of which are smooth). A morphism between smooth dynamical systems $\alpha=(f, F):(M, V) \rightarrow(N, W)$ is given by smooth maps, $f: M \rightarrow N$ and $F: T M \rightarrow T N$, such that the diagram

$\overline{1}$ This definition is a generalization of the one given in 9], although there it was defined as the category of dynamical systems and not smooth dynamical systems. 


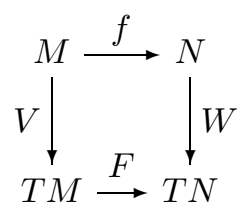

commutes, and for each $p \in M$ the restriction of $F$ to the fiber $T_{p} M,\left.F\right|_{T_{p} M}$ : $T_{p} M \rightarrow T_{f(p)} N$, is linear. In the case when $F$ is the pushforward of $f$, i.e., $F=f_{*}$, this definition implies that $V$ and $W$ are $f$-related (cf. [7]). A smooth dynamical subsystem is a pair $\left(S \subseteq M,\left.V\right|_{S}\right)$ where $S$ is an embedded submanifold of $M$ and $\left.V\right|_{S}$ is the restriction of a vector field $V$ on $M$ to $S$, and hence a vector field along $S$. As in the case of dynamical systems, morphisms of smooth dynamical subsystems are given in a way analogous to the definition of morphisms of dynamical systems (cf. 8] for a definition).

Note that there is a projection functor $\mathbf{P}_{\mathfrak{T o p}}: \mathfrak{D} \mathfrak{y} \rightarrow \mathfrak{T} \mathfrak{o p}$ from the category of dynamical systems to the category of topological spaces given by $\mathbf{P}_{\mathfrak{T o p}}(X, \varphi)=$ $X$ on objects and $\mathbf{P}_{\mathfrak{T} \mathfrak{p}}(h, r)=h$ on morphisms. Similarly, we have a projection functor from the category $\mathfrak{S} \mathfrak{d} \mathfrak{n}$ to the category $\mathfrak{M a n}, \mathbf{P}_{\mathfrak{M a n}}: \mathfrak{S} \mathfrak{d} \mathfrak{n} \rightarrow \mathfrak{M a n}$ defined in an analogous way.

Hybrid Systems. With the definitions of dynamical systems and smooth dynamical systems in hand, we can define hybrid systems. A classical hybrid system is a tuple $\mathscr{H}_{\text {class }}=\left(\mathbb{H}_{\text {class }}, \Phi\right)=(Q, E, D, G, R, \Phi)$ where $\mathbb{H}_{\text {class }}$ is a classical Hspace and $\Phi=\left\{\varphi_{i}\right\}_{i \in Q}$ where $\varphi_{i}$ is a flow on the topological space $D_{i}$, i.e., $\left(D_{i}, \varphi_{i}\right)$ is a dynamical system for each $i \in Q$.

Similarly, we can define smooth classical hybrid systems as pairs $\mathscr{G}_{\text {class }}=$ $\left(\mathbb{G}_{\text {class }}, V\right)=\left(Q, E, M, G^{M}, R^{S}, V\right)$ where $\mathbb{G}_{\text {class }}$ is a classical G-space and $V=$ $\left\{V_{i}\right\}_{i \in Q}$ where $V_{i}$ is a smooth vector field on the manifold $M_{i}$, i.e., $\left(M_{i}, V_{i}\right)$ is a smooth dynamical system for each $i \in Q$.

Definition 2. A categorical hybrid system is a pair $\mathscr{H}_{\text {cat }}=\left(\mathfrak{H}, \mathbf{S}_{\mathscr{H}}\right)$ where $\mathfrak{H}$ is an H-small category and $\mathbf{S}_{\mathscr{H}}: \mathfrak{H} \rightarrow \mathfrak{D} \mathfrak{y}$ is a functor such that the pair $\left(\mathfrak{H}, \mathbf{P}_{\mathfrak{T o p}} \circ \mathbf{S}_{\mathscr{H}}\right)$ is a categorical H-space. The H-space

$$
\mathbb{H}^{\mathscr{H}}=\left(\mathfrak{H}, \mathbf{P}_{\mathfrak{T} \mathfrak{o p}} \circ \mathbf{S}_{\mathscr{H}}\right):=\left(\mathfrak{H}, \mathbf{S}_{\mathbb{H}}^{\mathscr{H}}\right)
$$

is referred to as the underlying $\mathrm{H}$-space of the hybrid system $\mathscr{H}_{\text {cat }}$.

Theorem 2. If for each $e \in E$ there exists a morphism of dynamical subsystems

$$
\alpha_{e}:\left(G_{e} \subseteq D_{\mathfrak{s}(e)},\left.\varphi_{\mathfrak{s}(e)}\right|_{G_{e}}\right) \rightarrow\left(R_{e}\left(G_{e}\right) \subseteq D_{\mathfrak{t}(e)},\left.\varphi_{\mathfrak{t}(e)}\right|_{R_{e}\left(G_{e}\right)}\right),
$$

then there is an injective correspondence

$\left\{\right.$ Classical Hybrid Systems, $\left.\mathscr{H}_{\text {class }}\right\} \longrightarrow\left\{\right.$ Categorical Hybrid Systems, $\left.\mathscr{H}_{\text {cat }}\right\}$. 
Smooth Categorical Hybrid Systems. As in the case of categorical hybrid systems, we can define a smooth categorical hybrid system. A smooth categorical hybrid system is a pair $\mathscr{G}_{\text {cat }}=\left(\mathfrak{H}, \mathbf{T}_{\mathscr{G}}\right)$ where $\mathfrak{H}$ is an H-small category and $\mathbf{T}_{\mathscr{G}}: \mathfrak{H} \rightarrow \mathfrak{S} \mathfrak{d} \mathfrak{n}$ is a functor such that the pair $\left(\mathfrak{H}, \mathbf{P}_{\mathfrak{M a n}} \circ \mathbf{T}_{\mathscr{G}}\right)$ is a categorical G-space. As before, the underlying $G$-space of a smooth hybrid system $\mathscr{G}_{\text {cat }}$ is given by

$$
\mathbb{G}^{\mathscr{G}}=\left(\mathfrak{H}, \mathbf{P}_{\mathfrak{M a n}} \circ \mathbf{T}_{\mathscr{G}}\right):=\left(\mathfrak{H}, \mathbf{T}_{\mathbb{G}}^{\mathscr{G}}\right) .
$$

With this notation there is the following corollary of Theorem 2 .

Corollary 2. If for each edge $e \in E$ there exists a morphism of smooth dynamical subsystems

$$
\alpha_{e}:\left(G_{e} \subseteq M_{\mathfrak{s}(e)},\left.V_{\mathfrak{s}(e)}\right|_{G_{e}}\right) \rightarrow\left(R_{e}\left(G_{e}\right) \subseteq M_{\mathfrak{t}(e)},\left.V_{\mathfrak{t}(e)}\right|_{R_{e}\left(G_{e}\right)}\right),
$$

then there is an injective correspondence

\{Smooth Classical Hybrid Systems, $\left.\mathscr{G}_{\text {class }}\right\}$

$\left\{\right.$ Smooth Categorical Hybrid Systems, $\left.\mathscr{G}_{\text {cat }}\right\}$.

Remark 1. Because of Theorem $\pitchfork$ and $凤$ we use $\mathbb{H}$ and $\mathscr{H}$ to denote categorical hybrid spaces and hybrid systems, respectively, and simply refer to them as hybrid spaces and hybrid systems. Similarly, because of Corollary $\pitchfork$ and 2 we use $\mathbb{G}$ and $\mathscr{G}$ to denote smooth categorical hybrid spaces and systems; we simply refer to them as smooth hybrid spaces and systems.

The Categorical Framework for Hybrid Systems. To conclude this section, we note that the categorical framework introduced here gives a unifying framework for all of the definitions introduced here. More specifically, fixing an $\mathrm{H}$-small category $\mathfrak{H}$, an $\mathrm{H}$-space, G-space, hybrid system or smooth hybrid system is just given by the following functors

$$
\mathfrak{H} \stackrel{\mathbf{S}_{\mathbb{H}}}{\longrightarrow} \mathfrak{T} \mathfrak{o p}, \quad \mathfrak{H} \stackrel{\mathbf{T}_{\mathfrak{G}}}{\longrightarrow} \mathfrak{M a n}, \quad \mathfrak{H} \stackrel{\mathbf{S}_{\mathscr{H}}}{\longrightarrow} \mathfrak{D} \mathfrak{y n}, \quad \mathfrak{H} \stackrel{\mathbf{T}_{\mathscr{G}}}{\rightarrow} \mathfrak{S} \mathfrak{d} \mathfrak{n} \mathfrak{n},
$$

respectively. Namely, all that changes is the functor and the target category. Here the H-small category can be thought of as the "discrete" component of the hybrid system and the functor can be thought of as the "continuous" component. This general framework indicates that in studying hybrid systems, one need only consider functors from small categories to other categories. Note that this categorical notion of hybrid systems, hybrid spaces, et cetera, makes easy work of defining the category of hybrid systems and hybrid spaces. Studying the properties of these categories would seem to be a promising area of future research in hybrid systems. 


\section{$3 \quad$ Hybrid Homology}

In this section, a homology theory for hybrid systems is developed. Recall from Section 2 that every hybrid system has an underlying "space," termed an $\mathrm{H}$ space. With every H-space, we can associate a single topological space through the use of the homotopy colimit; the homology of this space is defined to be the hybrid homology of an H-space. Fortunately, there is a spectral sequence converging to the hybrid homology of an H-space. It will be seen that this spectral sequence implies a series of short exact sequences computing the hybrid homology in terms of the homology of a small category with coefficients in a certain functor. In the case when the H-space is contractible, the hybrid homology of this H-space is just the homology of a certain small category with coefficients in an abelian group.

The Homotopy Colimit. Let $\mathfrak{C}$ be a small category and $\mathbf{F}: \mathfrak{C} \rightarrow \mathfrak{T o p}$ a functor from this category to the category of topological spaces. There are two well known ways to associate to such a pair a single topological space. The first, and more obvious way, is through a construction known as the colimit. This is defined as

$$
\operatorname{colim}^{\mathfrak{C}}(\mathbf{F})=\frac{\coprod_{a \in \mathfrak{O} \mathfrak{b}(\mathfrak{C})} \mathbf{F}(a)}{x \sim \mathbf{F}(\alpha)(x)}, \quad \alpha \in \mathfrak{M o r}_{\mathrm{i}, d}(\mathfrak{C})
$$

This construction has been used in the past in hybrid systems, namely in [5], although it was not recognized that this was actually the colimit as the categorical definition of hybrid systems was not available in that paper; the hybrifold was defined as $\operatorname{colim}^{\mathfrak{H}}(\mathbf{S})$ for an $\mathrm{H}$-space $\mathbb{H}=(\mathfrak{H}, \mathbf{S}$ ) (for the rest of the paper we drop the "HH" subscript, i.e., we take $\left.\mathbf{S}=\mathbf{S}_{\mathbb{H}}\right)$. The key point is that although this construction is the obvious way of associating a single space to a hybrid system, it does not seem to be the "correct" one. There are many ways to justify this statement. In the context of algebraic topology, it has been known for a long time that the colimit does not possess desirable properties with respect to homotopies of spaces. A more subtle argument follows by considering the homology of these spaces; the colimit does not seem to encode the correct information about the behavior of hybrid systems - namely, with respect to Zeno. This problem is rooted in the fact that the colimit "forgets" about the information encoded in the edges of a hybrid system.

There is another, albeit more complicated, way of associating a single topological space to a hybrid system - through the homotopy colimit. This construction seems to encode the correct information about the hybrid system, both with respect to homotopies (cf. [1], [3]) and with respect to homology. For this reason we focus on the homotopy colimit. For simplicity, we will not introduce the definition of the homotopy colimit but refer the interested reader to [1] for a complete tutorial on homotopy colimits. The pertinent point regarding homotopy colimits is the simple form that they take when considering H-spaces. 


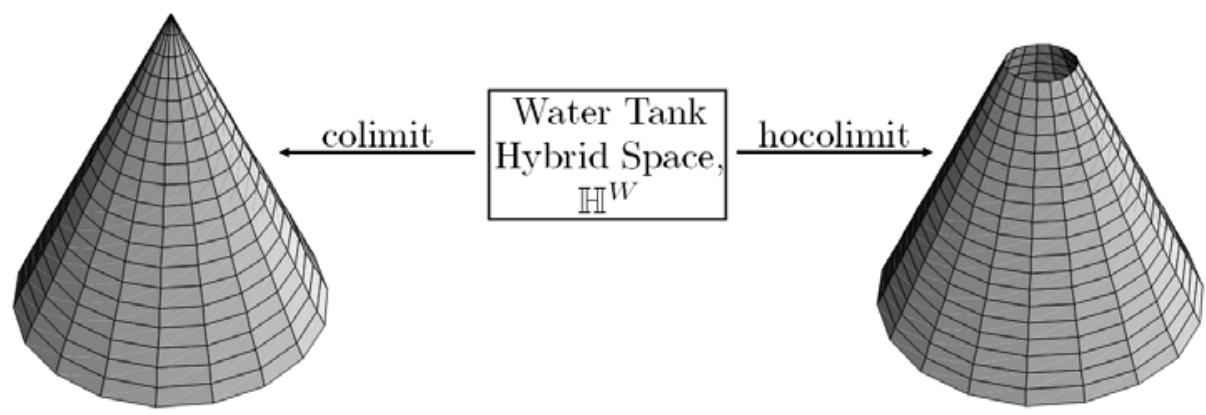

Fig. 1. The colimit and homotopy colimit of the water tank hybrid space

Theorem 3. For an $H$-space $\mathbb{H}=(\mathfrak{H}, \mathbf{S})$,

$$
\operatorname{hocolim}^{\mathfrak{H}}(\mathbf{S})=\frac{\left(\coprod_{b \in \vee(\mathfrak{H})} \mathbf{S}(b)\right) \amalg\left(\amalg_{a \in \wedge(\mathfrak{H})}(\mathbf{S}(a) \times I)\right)}{(x, 0) \sim \mathbf{S}\left(\alpha_{a}\right)(x),(x, 1) \sim \mathbf{S}\left(\beta_{a}\right)(x)} .
$$

Example 3. For the water tank hybrid space $\mathbb{H}^{W}=\left(\mathfrak{H}^{W}, \mathbf{S}^{W}\right)$, the colimit is homotopic to the (2-dimensional) cone, while the homotopy colimit is homotopic to the punctured cone (see Figure 1). It will be seen that the "hole" in this cone is a warning that the hybrid system may be Zeno, i.e., if the hole was not present, the hybrid system could not be Zeno - the topology of this space dictates the types of behavior this hybrid system can display.

The Underlying Topological Space of an H-Space. Since the homotopy colimit is a single topological space, we can define the underlying topological space of an $\mathrm{H}$-space as

$$
\operatorname{Top}(\mathbb{H}):=\operatorname{hocolim}^{\mathfrak{H}}(\mathbf{S})
$$

and we can consider the homology of this space. More explicitly, this gives a definition of hybrid homology - the homology of an H-space. The authors believe that this space will prove useful for other constructions on hybrid systems.

Definition 3. The homology of an $H$-space $\mathbb{H}=(\mathfrak{H}, \mathbf{S})$, denoted by $H H_{i}(\mathbb{H}, A)$ and termed the hybrid homology of $\mathbb{H}$ with coefficients in an abelian group $A$, is defined to be

$$
H H_{i}(\mathbb{H}, A):=H_{i}(\operatorname{Top}(\mathbb{H}), A)=H_{i}\left(\operatorname{hocolim}^{\mathfrak{H}}(\mathbf{S}), A\right) .
$$

The Homotopy Colimit Spectral Sequence. One of the important benefits of considering homotopy colimits is the homotopy colimit spectral sequence (cf. [1]) that relates the homology of the homotopy colimit to that of the homology of the underlying small category with coefficients in a functor. Note that there is not a similar spectral sequence for the colimit; this alone motivates the use of the 
homotopy colimit. Specifically, for a small category $\mathfrak{C}$ and functor $\mathbf{F}: \mathfrak{C} \rightarrow \mathfrak{T} \mathfrak{o p}$, there is a spectral sequence

$$
E_{p, q}^{2}=H_{p}\left(\mathfrak{C}, H_{q}(\mathbf{F}, A)\right) \Rightarrow H_{p+q}\left(\operatorname{hocolim}^{\mathfrak{C}}(\mathbf{F}), A\right) .
$$

Here $A$ is an abelian group and $H_{q}(\mathbf{F}, A): \mathfrak{C} \rightarrow \mathfrak{A} \mathfrak{b}$ is the functor from the small category to the category of abelian groups obtained by composing the homology functor $H_{q}(-, A): \mathfrak{T o p} \rightarrow \mathfrak{A b}$ with $\mathbf{F}$. The homology $H_{p}\left(\mathfrak{C}, H_{q}(\mathbf{F}, A)\right)$ is the homology of the small category $\mathfrak{C}$ with coefficients in the functor $H_{q}(\mathbf{F}, A)$. For a review of this homology theory, we refer the reader to [1, [10] and [1].

In the case of an $\mathrm{H}$-space $\mathbb{H}=(\mathfrak{H}, \mathbf{S})$ this spectral sequence gives us important information about the underlying topological space of the hybrid system, $\operatorname{Top}(\mathbb{H})$. In this case the spectral sequence becomes

$$
E_{p, q}^{2}=H_{p}\left(\mathfrak{H}, H_{q}(\mathbf{S}, A)\right) \Rightarrow H H_{p+q}(\mathbb{H}, A),
$$

and we refer to this spectral sequence as the hybrid homology spectral sequence. Because $\mathfrak{H}$ is an $\mathrm{H}$-small category, and by definition the longest chain of composable non-identity morphisms is of length one, for any functor $\mathbf{L}: \mathfrak{H} \rightarrow \mathfrak{A} \mathfrak{b}$,

$$
H_{n}(\mathfrak{H}, \mathbf{L})=0
$$

for $n \geq 2$. This implies that the spectral sequence will simplify even further into a set of short exact sequences.

Short Exact Sequences from a Spectral Sequence. Suppose that there is a spectral sequence $E_{p, q}^{2} \Rightarrow H_{p+q}$. If $E_{p, q}^{2}=0$ except when $p=0,1$ then there are short exact sequences

$$
0 \longrightarrow E_{1, n-1}^{2} \longrightarrow H_{n} \longrightarrow E_{0, n}^{2} \longrightarrow 0
$$

for all $n \geq 0$ (cf. [12]). Because $H_{n}(\mathfrak{H}, \mathbf{L})=0$ for $n \geq 2$ and any functor $\mathbf{L}: \mathfrak{H} \rightarrow$ $\mathfrak{A} \mathfrak{b}$, for the hybrid homology spectral sequence $E_{p, q}^{2}=H_{p}\left(\mathfrak{H}, H_{q}(\mathbf{S}, A)\right)=0$ for $p \neq 0,1$. Therefore, we have established the following important theorem.

Theorem 4. For an $H$-space $\mathbb{H}=(\mathfrak{H}, \mathbf{S})$ and an abelian group $A$, there are short exact sequences

$$
0 \longrightarrow H_{1}\left(\mathfrak{H}, H_{n-1}(\mathbf{S}, A)\right) \longrightarrow H H_{n}(\mathbb{H}, A) \longrightarrow H_{0}\left(\mathfrak{H}, H_{n}(\mathbf{S}, A)\right) \longrightarrow 0
$$

Collapsing Spectral Sequences. For a spectral sequence $E_{p, q}^{2} \Rightarrow H_{p+q}$, if $E_{p, q}^{2}=0$ except when $q=0$, then the spectral sequence is said to collapse. In this case there is an isomorphism $H_{n} \cong E_{n, 0}^{2}$. This isomorphism will yield the theorem shown below, which will be used in the following section to establish a very concrete method for computing the hybrid homology of an H-space in the case when the hybrid homology spectral sequence collapses. This will happen for a special class of hybrid systems, as given in the following definition. 
Definition 4. The H-space $\mathbb{H}=(\mathfrak{H}, \mathbf{S})$ is contractible if $\mathbf{S}(a)$ is contractible for every $a \in \mathfrak{O} \mathfrak{b}(\mathfrak{H})$ and $\mathbf{S}(\alpha) \sim$ id for every $\alpha \in \mathfrak{M o r}_{\mathfrak{i}, \alpha}(\mathfrak{H})$ (here $\sim$ denotes homotopic). We say that $\mathbb{H}$ is finite if $\mathfrak{H}$ has a finite number of objects and hence a finite number of morphisms. The H-space $\mathbb{H}$ is connected if Top(HH) is connected.

Theorem 5. If the H-space $\mathbb{H}=(\mathfrak{H}, \mathbf{S})$ is contractible, then

$$
H H_{i}(\mathbb{H}, A) \cong H_{i}(\mathfrak{H}, A)
$$

for an abelian group $A$. It follows that $H H_{n}(\mathbb{H}, A)=0$ for $n \geq 2$.

\section{Morse Theory and the Euler Characteristic of $\mathbf{H}$}

It is interesting to note that we can define the Euler characteristic for an $\mathrm{H}$-space $\mathbb{H}$. Moreover, it will be seen that the Euler characteristic of an $\mathrm{H}$-space can be expressed as a combination of the Euler characteristics of individual topological spaces in the hybrid space. As an application, a Morse theory type of theorem can be established for hybrid systems in a very special case.

The Euler Characteristic. Let $\mathbb{F}$ be a field. Since $\operatorname{Top}(\mathbb{H})$ is a topological space, we can define the Euler characteristic of the hybrid homology of an $\mathrm{H}$ space in the usual fashion. If $\operatorname{dim}_{\mathbb{F}} H H_{i}(\mathbb{H}, \mathbb{F})$ is finite and nonzero for only a finite number of $i^{\prime} s$ (here the dimension of $H H_{i}(\mathbb{H}, \mathbb{F})$ is its dimension as a vector space over $\mathbb{F})$, then the Euler characteristic of $\mathbb{H}$ with coefficients in a field $\mathbb{F}$ is given by

$$
\chi(\mathbb{H}, \mathbb{F})=\sum_{i=0}^{\infty}(-1)^{i} \operatorname{dim}_{\mathbb{F}} H H_{i}(\mathbb{H}, \mathbb{F}) .
$$

The Euler characteristic also can be defined when considering $H H_{i}(\mathbb{H})$; since this is not a vector space, the Euler characteristic is defined using the rank of an abelian group. For an abelian group $A$, define its $\operatorname{rank}($ over $\mathbb{Z})$ by $\operatorname{rank}_{\mathbb{Z}}(A)=$ $\operatorname{dim}_{\mathbb{Q}}\left(A \otimes_{\mathbb{Z}} \mathbb{Q}\right)$. In this case the Euler characteristic is defined to be

$$
\chi(\mathbb{H})=\sum_{i=0}^{\infty}(-1)^{i} \operatorname{rank}_{\mathbb{Z}} H H_{i}(\mathbb{H})=\sum_{i=0}^{\infty}(-1)^{i} \operatorname{dim}_{\mathbb{Q}} H H_{i}(\mathbb{H}) \otimes_{\mathbb{Z}} \mathbb{Q},
$$

where, again, for this to be well-defined, $H H_{i}(\mathbb{H}) \otimes_{\mathbb{Z}} \mathbb{Q}$ must be a finite dimensional vector space and nonzero for only a finite number of $i^{\prime} s$.

The main theorem of this section is that the Euler characteristic of an arbitrary $\mathrm{H}$-space can be computed in terms of the Euler characteristic of the topological spaces that determine the $\mathrm{H}$-space, i.e., the topological space $\mathbf{S}(a)$ for $a \in \mathfrak{O} \mathfrak{b}(\mathfrak{H})$. This theorem yields a corollary that allows for the easy computation of the Euler characteristic in a special case. 
Theorem 6. For an $H$-space $\mathbb{H}=(\mathfrak{H}, \mathbf{S})$,

$$
\chi(\mathbb{H}, \mathbb{F})=\sum_{a \in \mathfrak{O} \mathfrak{b}(\mathfrak{H})} \chi(\mathbf{S}(a), \mathbb{F})-\sum_{\alpha \in \mathfrak{M}_{\mathfrak{o r}} \mathfrak{r}_{\mathfrak{j} d}(\mathfrak{H})} \chi(\mathbf{S}(\mathfrak{s}(\alpha)), \mathbb{F})
$$

Corollary 3. For an $H$-space $\mathbb{H}=(\mathfrak{H}, \mathbf{S})$,

$$
\chi(\mathbb{H})=\sum_{a \in \mathfrak{O} \mathfrak{b}(\mathfrak{H})} \chi(\mathbf{S}(a))-\sum_{\alpha \in \mathfrak{M}_{\mathfrak{o r}} \mathfrak{r}_{\mathfrak{j} / \mathrm{d}}(\mathfrak{H})} \chi(\mathbf{S}(\mathfrak{s}(\alpha))) .
$$

If $\mathbb{H}$ is contractible and finite then for an arbitrary field $\mathbb{F}$

$$
\chi(\mathbb{H})=\chi(\mathbb{H}, \mathbb{F})=|\mathfrak{O} \mathfrak{b}(\mathfrak{H})|-\left|\mathfrak{M o r}_{\mathrm{i}, d}(\mathfrak{H})\right|
$$

where $|\mathfrak{O b}(\mathfrak{H})|$ is the number of objects of $\mathfrak{H}$ and $\left|\mathfrak{M o r}_{\mathrm{i}, d}(\mathfrak{H})\right|$ is the number of (non-identity) morphisms.

Morse Theory. The Euler characteristic is important because it relates the homology of a space with the behavior of flows on that space. It is possible to give a "Morse type theorem" for hybrid homology by considering the Morse theory of a smooth dynamical system.

Let $(M, V)$ be a smooth dynamical system as defined in Section 2, Assume that $M$ is a boundaryless compact $n$-dimensional manifold and that $V$ has only isolated singularities (equilibrium points). If $\operatorname{Index}(V)$ is the index of $V$, then the Poincaré-Hopf theorem states that

$$
\operatorname{Index}(V)=\chi(M) .
$$

Similarly, if $f$ is a Morse function on $M$, and $C(f)_{k}$ is the number of critical points of index $k$, then the Morse theorem says that

$$
\chi(M)=\sum_{k=0}^{n}(-1)^{k} C(f)_{k} .
$$

We will not review these definitions and constructions in this paper (for a complete review, the reader is referred to [13] and [14]). The important point is that it is possible to relate these results in smooth manifold theory to hybrid systems.

Now consider a smooth hybrid system $\mathscr{G}=\left(\mathfrak{H}, \mathbf{T}_{\mathscr{G}}\right)$ where $\mathbf{T}_{\mathscr{G}}: \mathfrak{H} \rightarrow \mathfrak{S d y n}$ and its corresponding underlying G-space $\mathbb{G}^{\mathscr{G}}=\left(\mathfrak{H}, \mathbf{P} \circ \mathbf{T}_{\mathscr{G}}\right):=\left(\mathfrak{H}, \mathbf{T}_{\mathbb{G}}^{\mathscr{G}}\right)$. Assume that for each object $a \in \mathfrak{O} \mathfrak{b}(\mathfrak{H}), \mathbf{T}_{\mathscr{G}}(a)=\left(M(a), X_{a}\right)$ where $M(a)$ is a smooth manifold and $X_{a}$ is a vector field on that manifold (this functor sends objects in $\mathfrak{H}$ to the subcategory of $\mathfrak{S} \mathfrak{d} \mathfrak{y} \mathfrak{n}$ whose objects are smooth dynamical systems), and that $M(a)$ is compact and boundaryless for every $a \in \mathfrak{O} \mathfrak{b}(\mathfrak{H})$. In this case call $\mathscr{G}$ a smooth compact boundaryless hybrid system.

Note that there is an embedding $\mathbf{E}: \mathfrak{G} \mathfrak{s p c} \rightarrow \mathfrak{H} \mathfrak{s p c}$ from the category of G-spaces, $\mathfrak{G} \mathfrak{s p c}$, to the category of H-spaces, $\mathfrak{H} \mathfrak{s p c}$ (cf. [8]). The underlying topological space of a smooth hybrid system is defined by $\operatorname{Top}(\mathbb{G}):=\operatorname{Top}(\mathbf{E}(\mathbb{G}))$, and 
we can consider the homology of these spaces, i.e., $H H_{i}(\mathbb{G}, A):=H H_{i}(\mathbf{E}(\mathbb{G}), A)$. Note that in general $\operatorname{Top}(\mathbb{G})$ is not a smooth manifold, or even a manifold at all. The amazing thing is that, regardless of this, it still is possible to obtain a Morse type theorem for hybrid systems of this form, i.e., we have the following corollary of Theorem 6 .

Corollary 4. Let $\mathscr{G}$ be a smooth compact boundaryless hybrid system and $\mathbb{G}^{\mathscr{G}}$ its underlying $G$-space. If $n(a)=\operatorname{dim}(M(a))$, then

$$
\begin{aligned}
& \chi\left(\mathbb{G}^{\mathscr{G}}\right)=\sum_{a \in \mathfrak{O} \mathfrak{b}(\mathfrak{H})} \operatorname{Index}\left(X_{a}\right)-\sum_{\alpha \in \mathfrak{M}_{\mathfrak{o r}} \mathfrak{r}_{\mathfrak{j} d}(\mathfrak{H})} \operatorname{Index}\left(X_{\mathfrak{s}(\alpha)}\right)
\end{aligned}
$$

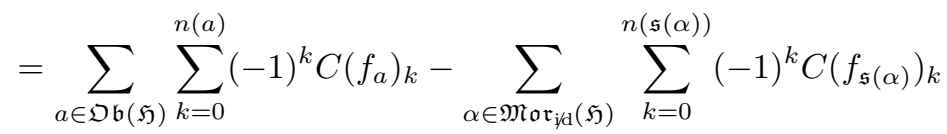

where $f_{a}$ is a Morse function of $M(a)$ for each $a \in \mathfrak{O} \mathfrak{b}(\mathfrak{H})$.

Remark 2. It would be desirable to determine a Morse type theorem involving only the topological space $\operatorname{Top}(\mathbb{G})$, but this does not seem possible (at least in any generality) because, as mentioned before, $\operatorname{Top}(\mathbb{G})$ is not a smooth manifold and will almost never be one - or even homeomorphic to one. Generalizations of this theorem seem most promising in the context of Conley index theory since those results are based on topological spaces and flows on those spaces.

\section{Characterization of Zeno Behavior Through Hybrid Homology}

In this section we show that the hybrid homology of an $\mathrm{H}$-space in some ways dictates the type of behavior that a hybrid system can have on this H-space. This result also will be related to the homology of the graph $\Gamma$ that a hybrid system has as its basic indexing set. Namely, we will show that in the case when the $\mathrm{H}$-space underlying a hybrid system is contractible, the vanishing of the hybrid homology in nonzero degrees implies that there are no Zeno executions. We will not review the definition of a hybrid system, or executions of hybrid systems; for a review of these definitions in the context of homology we refer the reader to [4]. Note that examples can also be found in this paper.

The Homology of a Graph. Recall that it is possible to define the homology of an oriented graph $\Gamma=(Q, E)$ with coefficients in the real numbers: $H_{i}(\Gamma, \mathbb{R})$. The important point about the homology of a graph is that it is easy to computeone need only compute the null space of the incidence matrix of the graph. For the graph $\Gamma$, the incidence matrix, denoted by $K_{\Gamma}$, is a $|Q| \times|E|$ matrix given by

$$
K_{\Gamma}=\left(\lambda_{\mathfrak{t}\left(e_{1}\right)}-\lambda_{\mathfrak{s}\left(e_{1}\right)} \cdots \lambda_{\mathfrak{t}\left(e_{|E|}\right)}-\lambda_{\mathfrak{s}\left(e_{|E|}\right)}\right)
$$

where $E=\left\{e_{1}, \ldots, e_{|E|}\right\}$ and $\lambda_{i}$ is the $i^{\text {th }}$ standard basis vector for $\mathbb{R}^{|Q|}$. 

then

It is easy to show (for a proof see [4]) that, if $\mathcal{N}\left(K_{\Gamma}\right)$ is the null space of $K_{\Gamma}$,

$$
H_{0}(\Gamma, \mathbb{R}) \cong \mathbb{R}^{|Q|-|E|+\operatorname{dim}_{\mathbb{R}} \mathcal{N}\left(K_{\Gamma}\right)}, \quad H_{1}(\Gamma, \mathbb{R}) \cong \mathbb{R}^{\operatorname{dim}_{\mathbb{R}} \mathcal{N}\left(K_{\Gamma}\right)}
$$

and $H_{n}(\Gamma, \mathbb{R})=0$ for $n \geq 2$. This implies that the Euler characteristic of $\Gamma$ is given by

$$
\chi(\Gamma)=\operatorname{dim}_{\mathbb{R}}\left(H_{0}(\Gamma, \mathbb{R})\right)-\operatorname{dim}_{\mathbb{R}}\left(H_{1}(\Gamma, \mathbb{R})\right)=|Q|-|E| .
$$

A Forgetful Functor. Given a small category $\mathfrak{C}$, we can "forget" about some of its structure in order to obtain a graph; in other words, there is a forgetful functor $\mathbf{U}: \mathfrak{C a t} \rightarrow \mathfrak{G} \mathfrak{r} \mathfrak{p h}$ where $\mathfrak{C a t}$ is the category of small categories and $\mathfrak{G} \mathfrak{r} \mathfrak{p h}$ is the category of small graphs. If $\mathfrak{C}$ is a small category, then the graph $\mathbf{U}(\mathfrak{C})$ is obtained by forgetting about which arrows are composites and which are identities; every functor $\mathbf{F}: \mathfrak{C} \rightarrow \mathfrak{C}^{\prime}$ is also a morphism $\mathbf{U}(\mathbf{F}): \mathbf{U}(\mathfrak{C}) \rightarrow \mathbf{U}\left(\mathfrak{C}^{\prime}\right)$ of graphs. For more details see [6].

It easily can be seen thaモthe category $\mathfrak{H} \mathfrak{c a t}$ of all $\mathrm{H}$-small categories is a full subcategory of the category $\mathfrak{C a t}($ cf. [8]). If $\mathbf{I}: \mathfrak{H} \mathfrak{c a t} \rightarrow \mathfrak{C a t}$ is the inclusion functor, then we have a functor from $\mathfrak{H} \mathfrak{c a t}$ to $\mathfrak{G} \mathfrak{r p h}$ given by the composition

$$
\mathfrak{H} \mathfrak{c a t} \stackrel{\mathrm{I}}{\longrightarrow} \mathfrak{C a t} \stackrel{\mathrm{U}}{\longrightarrow} \mathfrak{G} \mathfrak{r p h} .
$$

By abuse of notation, we will denote the composition of these two functors by $\mathbf{U}$ as well, i.e., $\mathbf{U}: \mathfrak{H} \mathfrak{c a t} \rightarrow \mathfrak{G r p h}$. This functor is important in that it relates the hybrid homology of an H-space to the homology of a graph.

Theorem 7. Let $\mathbb{H}=(\mathfrak{H}, \mathbf{S})$ be a finite and contractible H-space, then

$$
H H_{n}(\mathbb{H}, \mathbb{R}) \cong H_{n}(\mathbf{U}(\mathfrak{H}), \mathbb{R})
$$

where $H_{n}(\mathbf{U}(\mathfrak{H}), \mathbb{R})$ is the graph homology of the graph $\mathbf{U}(\mathfrak{H})$.

An important corollary of this theorem is that it gives a very easy and concrete way to compute the hybrid homology of a contractible and finite $\mathrm{H}$-space.

Corollary 5. Let $K_{\mathbf{U}(\mathfrak{H})}$ be the incidence matrix of the graph $\mathbf{U}(\mathfrak{H})$, then if $\mathbb{H}$ is contractible and finite

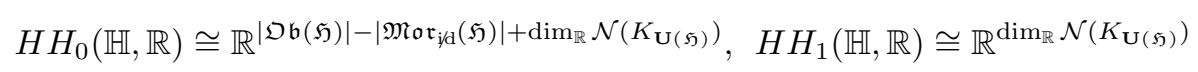

and $H H_{n}(\mathbb{H}, \mathbb{R})=0$ for $n \geq 2$.

Homological Relationships with Classical H-Spaces. If $\mathbb{H}=(\mathfrak{H}, \mathbf{S})$ is the (categorical) $\mathrm{H}$-space, obtained from the classical $\mathrm{H}$-space $\mathbb{H}_{\text {class }}=(\Gamma, D, G, R)$ via the correspondence given in Theorem 1, or vise versa, then we can relate these two "spaces" via homology — at least inthe case when $\mathbb{H}$ is contractible and finite. This relationship is given in the following proposition. This proposition supports the claim that the definition of a categorical $\mathrm{H}$-space is the right one because it says that when the domains of the hybrid system are contractible the hybrid homology of an $\mathrm{H}$-space is isomorphic to the graph homology. 
Proposition 1. Let $\mathbb{H}=(\mathfrak{H}, \mathbf{S})$ be the finite $H$-space obtained from the classical $H$-space $\mathbb{H}_{\text {class }}=(\Gamma, D, G, R)$. If $\mathbb{H}$ is contractible, then

$$
H H_{n}(\mathbb{H}, \mathbb{R}) \cong H_{n}(\Gamma, \mathbb{R})
$$

and it follows that $\chi(\mathbb{H})=\chi(\Gamma)$.

A rather startling point is that the underlying H-space of a hybrid systemmore specifically its homology - in some way dictates the behavior that this hybrid system can display (for a complete discussion on this, as well examples and a review of Zeno behavior, see [4]). Even more importantly, the type of behavior that the homology of an H-space "notices" is exactly the behavior that is central, and unique, to hybrid systems: Zeno behavior. This point is made more clear in the following theorem:

Theorem 8. Let $\mathbb{H}^{\mathscr{H}}=\left(\mathfrak{H}, \mathbf{S}^{\mathscr{H}}\right):=\left(\mathfrak{H}, \mathbf{P}_{\mathfrak{T} \mathfrak{o p}} \circ \mathbf{S}_{\mathscr{H}}\right)$ be the underlying H-space of the hybrid system $\mathscr{H}=\left(\mathfrak{H}, \mathbf{S}_{\mathscr{H}}\right)$. If $\mathbb{H}^{\mathscr{H}}$ is contractible and finite, then

$$
\operatorname{dim}_{\mathbb{R}} H H_{1}\left(\mathbb{H}^{\mathscr{H}}, \mathbb{R}\right)=\operatorname{dim}_{\mathbb{R}} \mathcal{N}\left(K_{\mathbf{U}(\mathfrak{H})}\right)=0 \quad \Rightarrow \quad \mathscr{H} \text { is not Zeno. }
$$

If $\mathbb{H}^{\mathscr{H}}$ is connected, it implies that $\operatorname{dim}_{\mathbb{R}} H H_{0}\left(\mathbb{H}^{\mathscr{H}}, \mathbb{R}\right)=1$, and so we have the following corollary to this theorem which is in a form more reminiscent of "Morse-type" theorems.

Corollary 6. If $\mathbb{H}^{\mathscr{H}}$ is connected, contractible and finite, then

$$
\chi\left(\mathbb{H}^{\mathscr{H}}\right)=|\mathfrak{O} \mathfrak{b}(\mathfrak{H})|-\left|\mathfrak{M o r}_{\mathrm{i}, \mathrm{d}}(\mathfrak{H})\right|=1 \quad \Rightarrow \quad \mathscr{H} \text { is not Zeno. }
$$

In many ways, this theorem (and its corollary) is more of a "Morse-type" theorem than Theorem 4. The hope is, through the use of the categorical framework for hybrid systems introduced here, to incorporate the dynamics of a hybrid system into the above theorems in order to obtain tighter algebraic theorems on the nonexistence of Zeno.

Example 4. For the water tank hybrid space $\mathbb{H}^{W}$, using Proposition 1, it is easy to see that $H H_{1}\left(\mathbb{H}^{W}, \mathbb{R}\right) \cong H H_{0}\left(\mathbb{H}^{W}, \mathbb{R}\right) \cong \mathbb{R}$. So we cannot say that the water tank is not Zeno, which is good because it is Zeno.

\section{References}

1. Bousfield, A.K., Kan, D.M.: Homotopy Limits, Completions and Localizations. Volume 304 of Lecture Notes in Mathematics. Springer-Verlag (1972)

2. Thomason, R.W.: First quadrant spectral sequences in algebraic $K$-theory. In Dupont, J.L., Madsen, I.H., eds.: Algebraic Topology. Volume 763 of Lecture Notes in Mathematics. Springer-Verlag (1978) 332-355

3. Vogt, R.M.: Homotopy limits and colimits. Mathematische Zeitschrift 134 (1973) $11-52$

4. Ames, A.D., Sastry, S.: Characterization of Zeno behavior in hybrid systems using homological methods. Submitted to ACC (2005) 
5. Simic, S., Johansson, K.H., Sastry, S., Lygeros, J.: Towards a geometric theory of hybrid systems. In Krogh, B., Lynch, N., eds.: HSCC. Volume 1790 of LNCS., Springer Verlag (2000) 421-436

6. Lane, S.M.: Categories for the Working Mathematician. second edn. Volume 5 of Graduate Texts in Mathematics. Springer (1998)

7. Lee, J.M.: Introduction to Smooth Manifolds. Volume 218 of Graduate Texts in Mathematics. Springer (2003)

8. Ames, A.D., Sastry, S.: A categorical theory of hybrid systems. Technical Report (2004)

9. Haghverdi, E., Tabuada, P., Pappas, G.J.: Bisimulation relations for dynamical, control, and hybrid systems. Submitted to Theoretical Computer Science (2003)

10. Gabriel, P., Zisman, M.: Calculus of Fractions and Homotopy Theory. Volume 35 of Ergenbnisse der Mathematik und Ihrer Grenzgebiete. Springer-Verlag (1967)

11. Thomason, R.W.: First quadrant spectral sequences in algebraic $K$-theory via homotopy colimits. Communications in Algebra 10 (1982) 1589-1668

12. Weibel, C.A.: An Introduction to Homological Algebra. Cambridge University Press (1994)

13. Milnor, J.: Morse Theory. Princeton University (1963)

14. Madsen, I., Tornehave, J.: From Calculus to Cohomology: De Rahm Cohomology and Characteristic Classes. Cambridge University (1997) 